Elsevier Editorial System(tm) for Colloids and Surfaces A: Physicochemical and Engineering Aspects

Manuscript Draft

Manuscript Number:

Title: The Interactions of fibres with soap films

Article Type: SI: Eufoam 2016

Keywords: Fibre

Foam

Soap films

Surface Evolver

Corresponding Author: Dr. Benjamin Haffner,

Corresponding Author's Institution:

First Author: David Whyte

Order of Authors: David Whyte; Benjamin Haffner; Atsushi Tanaka; Tuomo Hjelt; Stefan Hutzler

Abstract: We present experiments and Surface Evolver simulations concerning the interaction of fibres with simple arrangements of soap films, which constitute model systems for dry foams. For a fibre inserted into a soap film which spans two Plateau borders, our simulation accurately predicts the variation in the length of the film as the fibre thickness is varied. For a fibre introduced into a Plateau border, simulations accurately predict the variation in length of the Plateau border

as the fibre diameter is varied, and as it is moved. We suggest that the force necessary to move the fibre from equilibrium may act to inhibit foam coarsening, in line with observations from experiments on fibreladen foams. 


\section{Trinity College Dublin}

Coláiste na Tríonóide, Baile Âtha Cliath The University of Dublin

EUFOAM Proceedings Office

Dublin, 28/10/2016

\section{Dear Editor,}

Please find enclosed our manuscript entitled: "Interactions of fibres with simple arrangements of soap films" which was presented in the last EUFOAM Conference by David Whyte. This work presents an experimental investigation of the configuration of soap films in the presence of fibre. The data are described both theoretically and numerically. The very good description of our experimental data with our simulations allow to predict the configuration of the films as a function of the features of the fibre.

In addition, our observations raise the question of the interaction between foam and fibres at the macroscopic scale, this appears as a promising topic, in particular for industry dealing with novel materials. Thus, we believe our observations should interest the broad community of researcher in fluid mechanics working on foam containing solid material. We therefore would be very excited to see this work published in the EUFOAM Proceedings, which we believe reaches the most relevant audience for our work.

Sincerely yours,

David Whyte, Benjamin Haffner, Atsushi Tanaka, Tuomo Hjelt and Stefan Hutzler 


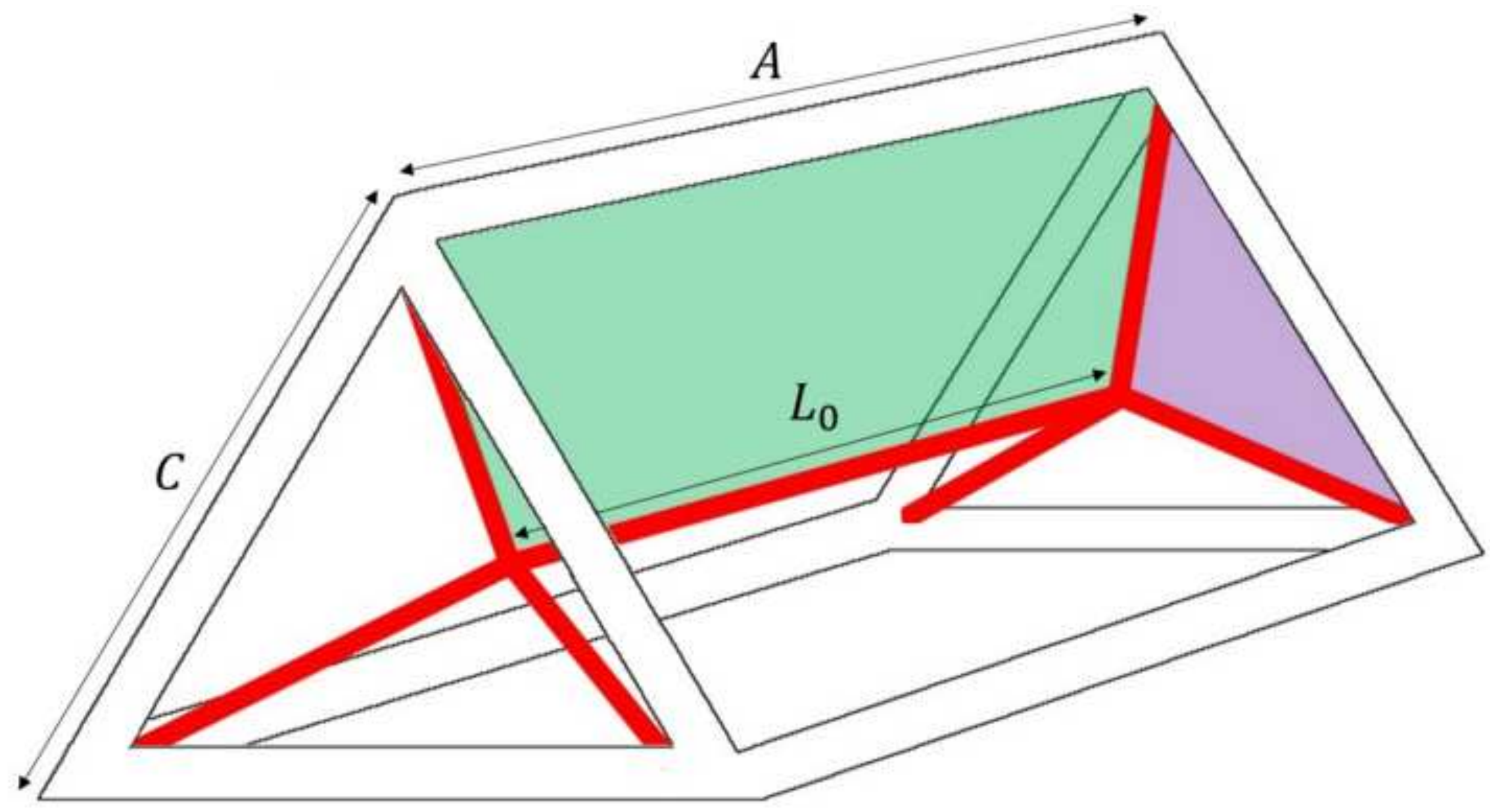

After dipping the frame (equilateral triangular prism, aspect ration $\mathrm{a}=\mathrm{A} / \mathrm{C}$ ) in the surfactant solution, a Plateau border of length $\mathrm{L}_{0}$ is created along with nine soap films.

This set-up allows to study the interraction between a fibre and a single Plateau border. 


\section{Highlights}

Our manuscript entitled: "Interactions of fibres with simple arrangements of soap films" was presented in the last EUFOAM Conference by David Whyte. This work proposes an experimental investigation of the configuration of soap films in the presence of fibre. The data are described both theoretically and numerically, indeed, geometrical considerations outline the problem in the simplest case and Surface Evolver simulations describe the more complicated cases. Responses on the stability of films containing fibre have been highlighted and the role of the fibre shape, fibre thickness as much as the fibre position on films deformation are now known. The excellent description of our experimental data with our simulations allow to predict the configuration of the films as a function of the features of the fibre. This work finds relevance in multiple applications involving fibres such as in papermaking where the use of foam along with fibres appears as a new way of reducing cost productions, concerning building materials, the acoustic insulation requires particularly low density. 


\title{
Interactions of fibres with simple arrangements of
} soap films

\author{
David Whyte ${ }^{1}$, Benjamin Haffner ${ }^{1}$, Atsushi Tanaka² ${ }^{2}$ Tuomo Hjelt ${ }^{2}$, \\ and Stefan Hutzler ${ }^{1}$ \\ ${ }^{1}$ School of Physics, Trinity College Dublin, The University of Dublin, \\ Ireland \\ ${ }^{2}$ VTT Technical Research Centre of Finland Ltd, Finland
}

October 28, 2016

\begin{abstract}
We present experiments and Surface Evolver simulations concerning the interaction of fibres with simple arrangements of soap films, which constitute model systems for dry foams. For a fibre inserted into a soap film which spans two Plateau borders, our simulation accurately predicts the variation in the length of the film as the fibre thickness is varied. For a fibre introduced into a Plateau border, simulations accurately predict the variation in length of the Plateau border as the fibre diameter is varied, and as it is moved. We suggest that the force necessary to move the fibre from its equilibrium position may act to inhibit foam coarsening, in line with previous observations from experiments on fibre-laden foams.
\end{abstract}




\section{Introduction}

In the paper making industry, foam forming is a relatively new manufacturing technique in which the suspension of cellulose fibres in water is foamed before drying it to form paper [1]. The bubbles act to space out the fibres more evenly, improving the homogeneity and the strength of the final product. For this reason, foam forming is of great advantage to the papermaking industry. Related is the use of this technique for the production of novel non-woven fibrous material for thermal insulation. Although the technique has been known since 1974 [1] it has been only very recently that the interactions between fibres and foam have been examined in greater detail. $[2,3,4,5,6]$.

Adding fibres to a liquid foam alters its physical properties (e.g. drainage rate, bubble size distribution, coarsening rate) significantly. Recent work [2, $3,4]$ concerned mainly wet foams, i.e. those with liquid volume fraction $\phi \gtrsim$ 0.2 , and focused on the effect of the presence of fibres on global properties such as stability of the foam [5] or rheology [7] of fibre suspensions.

In this paper we consider the local effects of fibres on configurations of soap films. We present two experiments probing the interaction of a single fibre with the simplest possible model systems for dry foams: one essentially $2 \mathrm{D}$ in character, the other fully 3D. We compare data obtained from these experiments to that obtained from simulations using the Surface Evolver software package [8].

Finally we suggest that this fibre-film interaction plays a role in the observed reduction of coarsening rate in fibre-laden foams. 


\section{The effect of fibres on soap films between two parallel plates}

Soap films, under the action of surface tension, attempt to minimize their total surface area. The resultant structures obey Plateau's laws $[9,10]$ :

1. Soap films are smooth surfaces of constant mean curvature.

2. Soap films meet in threes at angles of $120^{\circ}$ at liquid channels called Plateau borders.

3. Plateau borders meet in fours at angles of $\sim 109^{\circ}$ at vertices.

To these is often added [11]:

4. Soap films meet boundaries at $90^{\circ}$.

These laws apply to dry foams in equilibrium.

In two dimensions these rules are related to the Steiner problem: finding the shortest possible length of lines linking a set of points. Solutions to the Steiner problem consist of sets of straight lines meeting in threes at angles of $120^{\circ}$, analogous to Plateau's second law. The solution for the corners of a square is shown in Fig. 1(a). Simple geometry gives the length $L_{0}$ of the central line as

$$
\frac{L_{0}}{S}=1-\frac{1}{\sqrt{3}} \approx 0.42,
$$

where $S$ is the side length of the square.

Fig. 1(b) shows an experimental apparatus consisting of two parallel Perspex plates bridged by four graphite pins arranged in a square. We have used this to investigate how the addition of a fibre changes this equilibrium configuration of soap film. The apparatus is dipped in a surfactant solution, the network of films formed between the pins is a Steiner tree of the same form as Fig. 1(a). Since the films are parallel to the two perspex plates, we call this a quasi-2D apparatus. 


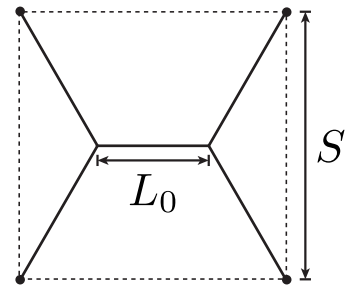

(a)

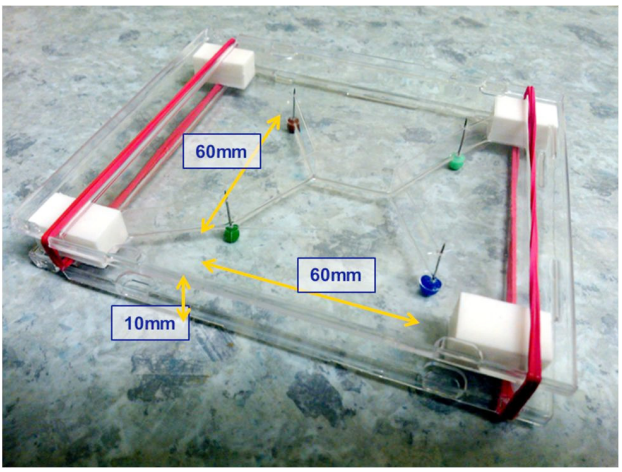

(b)

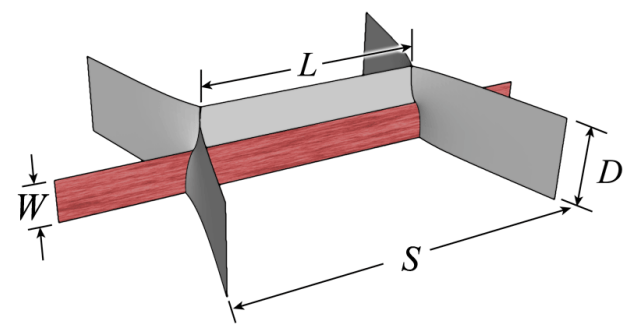

(c)
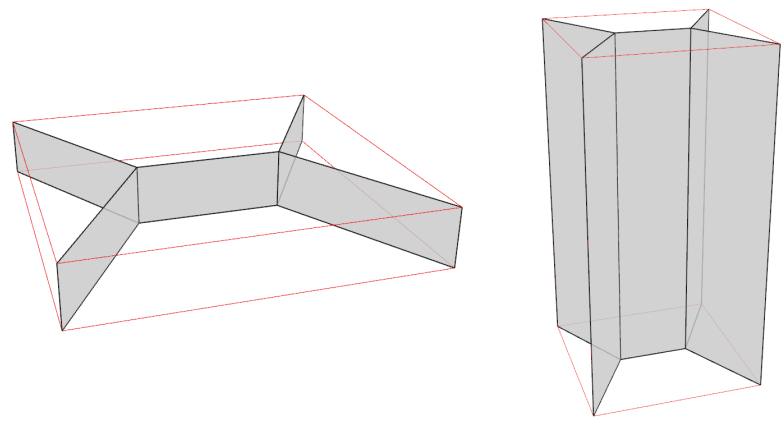

(d)

Figure 1: (a) The shortest possible network of lines connecting four points arranged in a square has lines meeting in threes at $120^{\circ}$ (Steiner problem). (b) Two Perspex plates bridged by graphite pins after being dipped in a soap solution yields the corresponding quasi-2d arrangement of soap films. We use this apparatus to probe for the effect of fibres introduced into the plane of the central film, as shown in c) for a fibre of width $W$. The length $L$ of the film is measured on the top plate, as shown here. The plates are separated by a distance $D$. (d) Examples of asplect ratios used in our experiments. 
In our experiments (Fig. 1b) we place a flat wooden stick (thickness of $1 \mathrm{~mm}$ ) into the plane of the central film and measure the variation in the length $L$ of the film (as viewed on the top), for various fibre widths $W$. Fig. 1c shows the definitions of the relevant lengths (i.e. the pin separation $S$, the plate separation $D$, the fibre width $W$ and the length of the central film $L$ ) for our setup. Since the liquid films meet the fibre at right angles (Plateau's 4th rule) this results in an increase in the length $L$ as compared to $L_{0}$, its length in the absence of a fibre. The experiments were repeated for three different aspect ratios $D / S$, see Fig. 1d.

The cases $W=0$ (i.e. no fibre) and $W=D$ (fibre spanning the space between the two plates) result in $L=L_{0} \approx 0.42 S$ (Steiner problem) and $L=S$ (from Plateaus 4th rule), respectively. This is regardless of the aspect ratio $D / S$ of the set-up.

Fig. 2 shows measured values for the variation of $L / S$ with $W / D$. The relationships are highly non-linear with a strong dependence also on the different aspect ratios of $D / S$. Also shown are results from computer simulations using the Surface Evolver program [8], a software which has been employed previously to model similar soap film configurations $[12,13]$ (Details of the simulation are left to Appendix A). For all three aspect ratios used experimentally, our simulation accurately predicts the variation of film length with fibre thickness. Also the respective values of $L$ in the limits $W=0$ and $W=D$ are reproduced.

Our experimental and numerical data (Fig. 2) is well approximated by the empirical equation

$$
\frac{L}{S}=k_{1}+k_{2} \frac{\exp (\beta W / D)-1}{\exp (\beta)-1},
$$

with one free parameter $\beta$, and constants $k_{1}=1-1 / \sqrt{3}$ and $k_{2}=1 / \sqrt{3}$. The theoretical endpoints are fixed by this form, and in the limit $\beta \rightarrow 0$ we recover a straight line between these endpoints. In Fig. 3 (a) we show such 


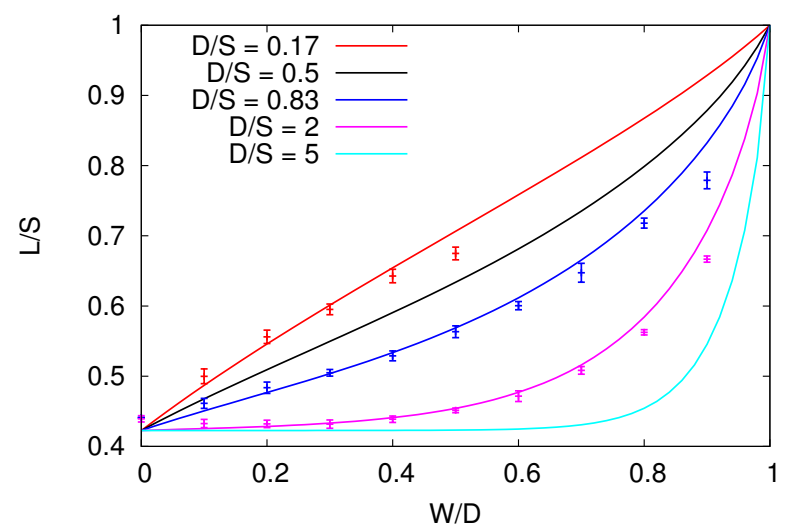

Figure 2: As we increase the fibre thickness $W$ from 0 to its maximum value of $D$, the length $L$ of the film, as measured on the top plate, increases from $\sim 0.42$ to 1 . The shape of the curve between these endpoints depends strongly on the aspect ratio $D / S$. Our experimentally measured data points are well described by our Surface Evolver simulations, represented as solid lines.

fits to our Surface Evolver data for three different values of $D / S$. The inset shows that the parameter $\beta$ increases approximately linear with aspect ratio $D / S$, i.e. $\beta \simeq \alpha D / S$, with a fitting constant $\alpha \simeq 3.19$. We thus arrive at the following empirical relationship for the variation of central film length (as viewed from the top),

$$
L / S=k_{1}+k_{2} \frac{\exp (\alpha W / S)-1}{\exp (\alpha D / S)-1}
$$

which is plotted in Fig. 3 (b).

\section{The effect of fibres on a single Plateau border}

Having shown that our Surface Evolver simulations are successful in modelling the altered film configuration due to the presence of fibres in a quasi-2d set-up, we will now turn to the influence of a long fibre on a simple $3 \mathrm{~d}$ film 


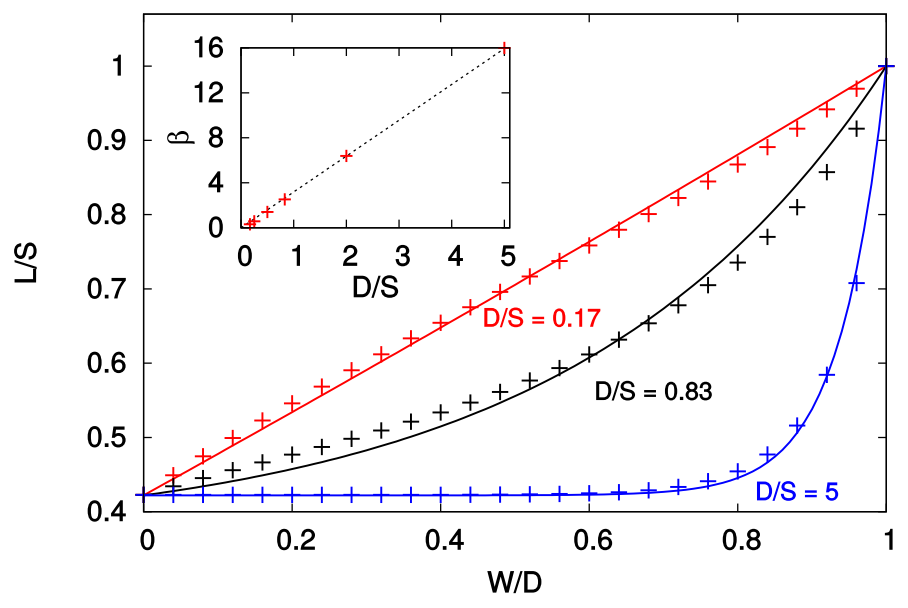

(a)

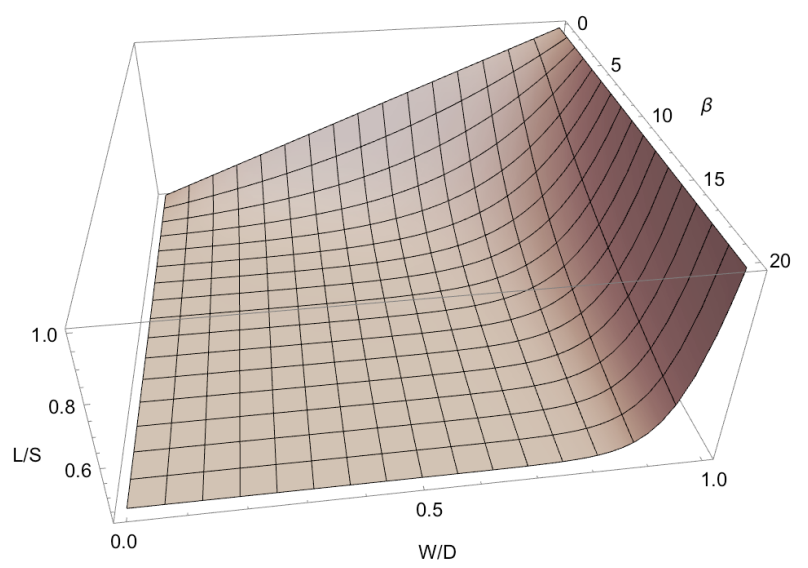

(b)

Figure 3: (a) For various aspect ratios $D / S$, the variation of the film length with fibre thickness, as obtained from Surface Evolver simulations (crosses) can be approximated by the empirical form (Eqn. (2)) (solid lines), with only one free parameter $\beta$. These curves represent, $\beta \approx 0.3,2.5,16$ respectively. The inset shows that this fit parameter $\beta$ increases approximately linearly with $D / S$. (b) 3-D plot showing the variation of film length $L$ as a function of fibre width $W$ and $\beta=3.19 D / S$, as computed from eq.(3). 


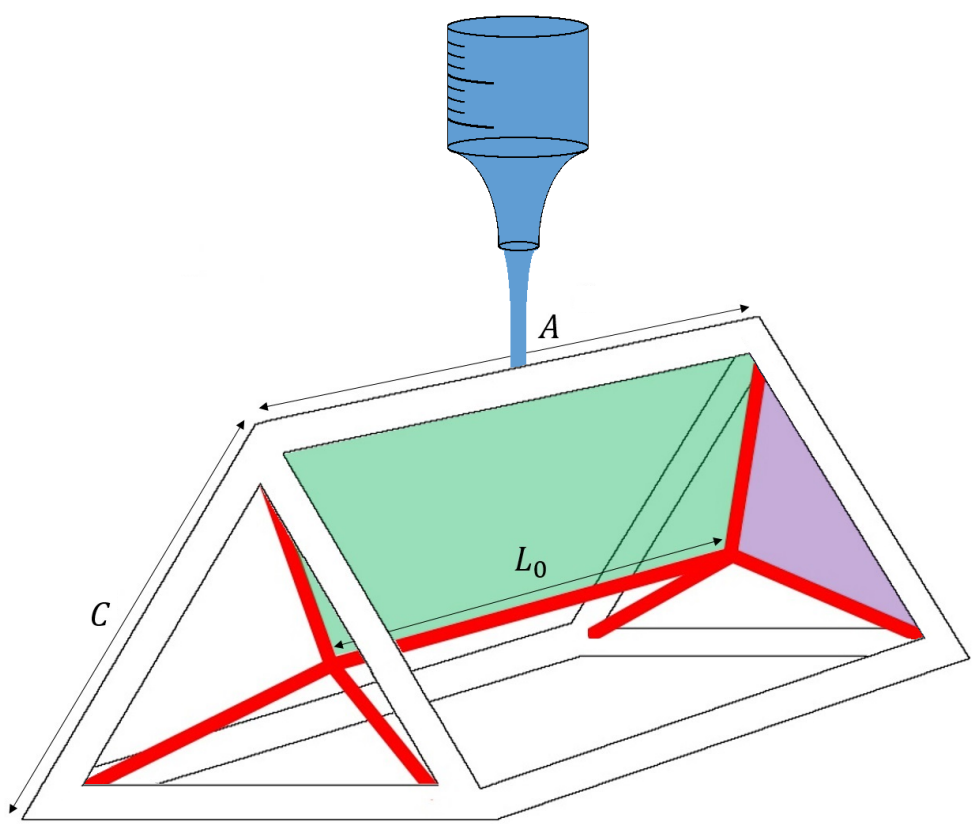

Figure 4: In our experiments we feed a frame (equilateral triangular prism) from the top with surfactant solution, using a burette. The edges $A$ and $C$ allow to define the aspect ratio $a=A / C$. After dipping the frame in the surfactant solution, a Plateau border of length $L_{0}$ is created along with nine soap films. In green we show one of the three trapezoid-shaped films, in purple, one of the six triangle-shaped films.

configuration. Such configurations may be obtained by withdrawing wire frames from a surfactant solution. Indeed it was this type of experiments that lead Joseph Plateau to the formulation of the equilibrium rules for soap films [9], as stated in Section 2. More recently, a cubic wire frame was used to investigate the stability of eightfold vertices in microgravity [13].

When a wire frame in the shape of an equilateral triangular prism (see Fig. 4) is dipped into a surfactant solution (in our case commercial washingup liquid Fairy, $3 \mathrm{~g} / \mathrm{L}$ ), a single central Plateau border is formed, connecting two fourfold Plateau border junctions, similarly to Plateau borders in a dry bulk foam. 


\begin{tabular}{cc} 
Aspect ratio $a$ & Height $C$ of prism $(\mathrm{mm})$ \\
\hline 0.86 & 31 \\
1.25 & 45 \\
1.5 & 69 \\
2.89 & 104 \\
\hline
\end{tabular}

Table 1: Relevant dimensions of the wire frames used in our experiments.

In our experiments we feed the wire frame from the top with a burette containing the surfactant solution (flow rate $\approx 0.25 \mathrm{~mL} / \mathrm{min}$ ) to prevent thinning of the films and hence their breakup. (In our experiments we were able to maintain the films for more than 10 minutes.) Plateau border lengths were measured using Image J sofware [14] from side-view pictures taken from a Canon EOS 50D camera.

From here on we will normalize lengths by the length of the triangular side $C$ of the frame (see fig 4 ), defining our aspect ratio $a=A / C$ and a normalized Plateau border length $l_{0}=L_{0} / C$, where $L_{0}$ is the length of the central Plateau border in the absence of fibres. In total, five frame aspect ratio have been used for the experiments (see Table 1).

According to Plateau's third rule (section 2), the Plateau borders meet at angles of $\arccos (-1 / 3) \approx 109^{\circ}$. Hence via simple geometry we can determine the normalized length $l_{0}$ of the central Plateau border as a function of the aspect ratio $a$,

$$
l_{0}=a-\frac{1}{\sqrt{6}} \approx a-0.41 .
$$

Before turning to the effect of fibres on the film geometry we have tested the accuracy of both our experiments and Surface Evolver simulations. Fig. 5 shows $l_{0}$ for a variety of aspect ratios $a$ in excellent agreement with eqn. (4). The Surface Evolver values are all accurate to the theoretical values to within $10^{-5}$, and the experimental data agrees within experimental 


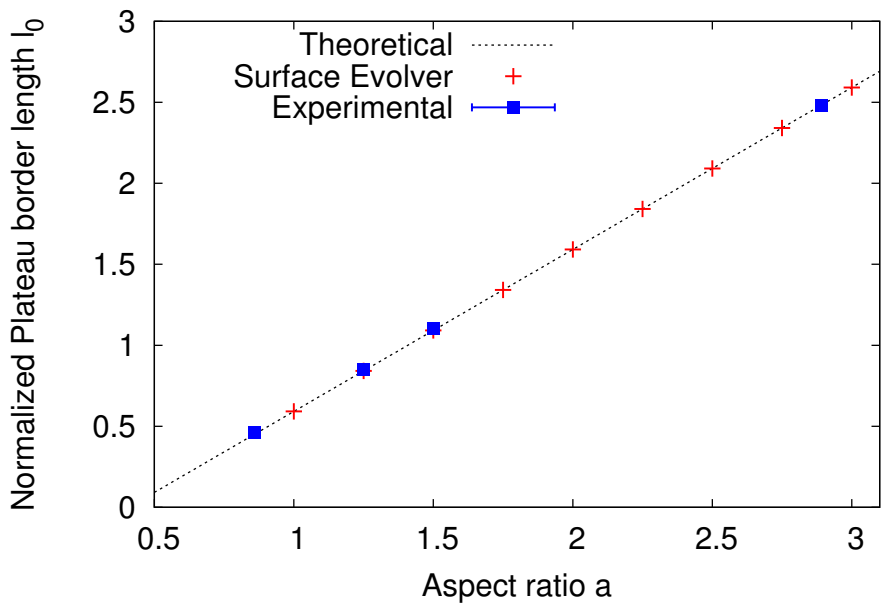

Figure 5: Length of central Plateau border in an equilateral triangular prism. The normalized Plateau border length $l_{0}$ as a function of the frame aspect ratio $a$, as obtained experimentally and from simulation using the Surface Evolver, is in excellent agreement with the analytical result, eqn.(4). The error bars for the experimental data are too small to be visible here. 
error with theory.

\subsection{Variation of surface Plateau border length with fibre di- ameter}

When a cylindrical fibre is introduced into the central Plateau border, as shown in Fig. 6(a) for experiments and Fig. 6(b) for simulations, three surface Plateau borders form where the three films meet the fibre.

Surface Plateau borders meet Plateau borders at angles of $90^{\circ}$, rather than $109^{\circ}$. Hence the length of these surface Plateau borders (once again, normalized by the side length $C$ of the triangle), which we denote $l$, is greater than the length $l_{0}$ of the original central Plateau border. This increase in length increases with fibre thickness (see fig 6 (c)).

We find that the surface Plateau border length $l$ depends on the normalized diameter $d$ of the fibre which is introduced into the central Plateau border, as well as on the aspect ratio $a$ of the frame. However, we can eliminate the effects of varying aspect ratios by considering the quantity $\Delta l=l-l_{0}$, i.e. the increase in the length of the Plateau border compared to the length of the Plateau border in the absence of a fibre.

In Fig. 7 we plot $\Delta l$ as a function of $d$, as obtained from experiments using a variety of frame aspect ratios, fibre diameters and materials (see

Table 2). In addition we show results from Surface Evolver simulations, details of which are given in Appendix A.

Within experimental error, our simulations correctly predict the length of the surface Plateau borders.

\subsection{Variation of Plateau border length with fibre position}

We now wish to investigate the behaviour of the surface Plateau borders as the fibre is slowly displaced from its central position. To avoid having to deal with an overwhelming number of variables, from here on we use a steel frame with $C=46 \mathrm{~mm}$ and $A=69 \mathrm{~mm}$, and use a fibre of diameter $1 \mathrm{~mm}$, 


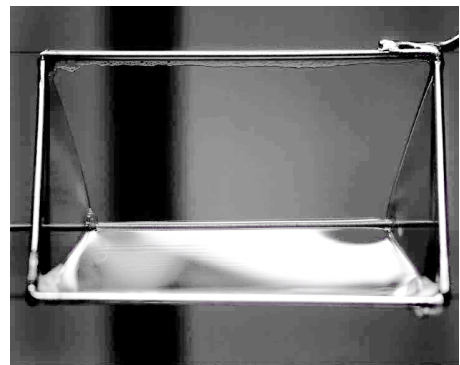

(a)

(c)

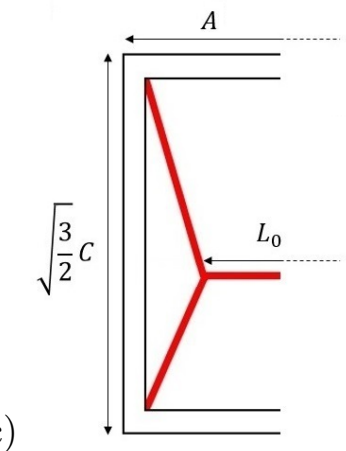

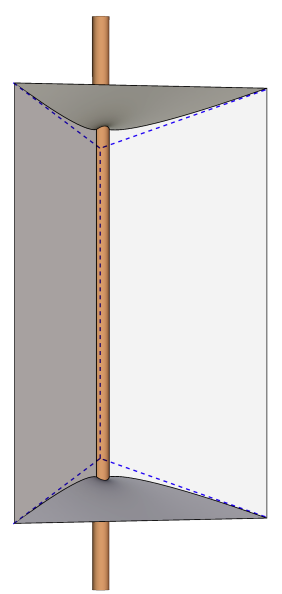

(b)

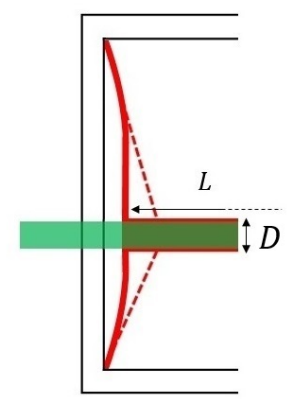

Figure 6: (a) Photograph of our experimental setup; a cylindrical fibre made of copper with a diameter of $1 \mathrm{~mm}$ has been introduced into the central Plateau border (frame length $\mathrm{C}=69 \mathrm{~mm}$ ). (b) In our simulation using the Surface Evolver one can see that the surface Plateau borders on the fibre are longer than the original Plateau border length (dashed lines). (c) This sketch of the left vertex shows the Plateau border without fibre (left), containing a thin fibre (middle), and a thick fibre (right). The Plateau border length increases with the diameter $D$ of the fibre. 


\begin{tabular}{rc} 
Material & Diameter $D(\mathbf{m m})$ \\
\hline Steel & 0.55 \\
& 1.5 \\
& 1.6 \\
& 2 \\
& 2.1 \\
\hline Plastic & 1.1 \\
& 3 \\
& 3.4 \\
\hline Copper & 0.8 \\
& 1 \\
\hline Solder & 1 \\
& 2.1 \\
\hline
\end{tabular}

Table 2: Materials and diameters of the fibres used in our experiments.

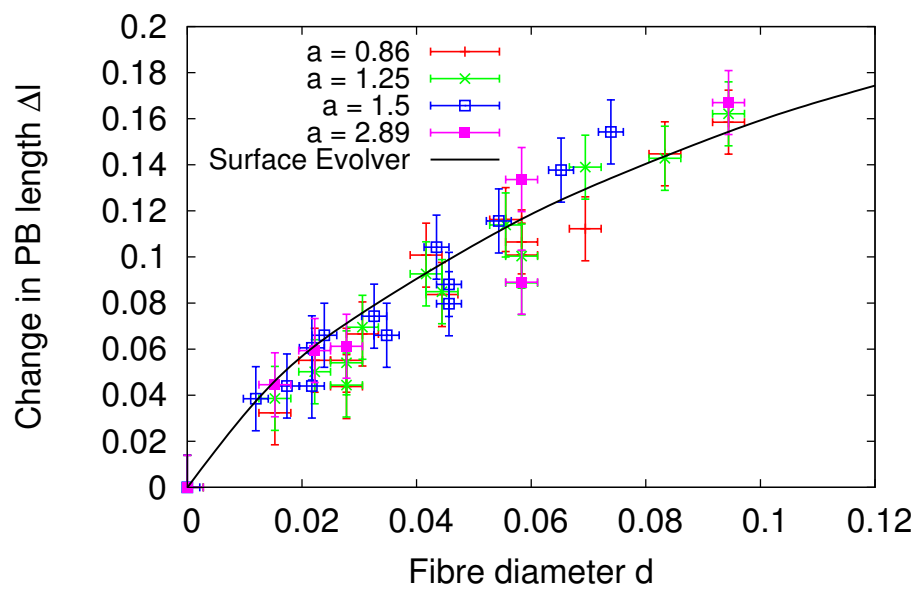

Figure 7: As fibres with diameter $d$ are introduced into the central Plateau border, its length increases by an amount $\Delta l$. Surface Evolver simulations (line) accurately predict the variation of $\Delta l$ with $d$, as found experimentally for various aspect ratios $a=A / C$. 
giving $a=1.5$, and $d \approx 0.022$. Initially we will consider moving the fibre along the axis of symmetry of the equilateral triangle by a distance $x$, as shown in Fig. 8(a).

Moving the fibre breaks the threefold rotational symmetry, and so the surface Plateau borders are not of equal length. We denote the two different surface Plateau border lengths (normalized as before by side length $C$ ) by $l_{1}$ and $l_{2}$. Similarly to before, we define $\Delta l_{1}=l_{1}-l_{0}$ and $\Delta l_{2}=l_{2}-l_{0}$ to denote their respective increases relative to the length of the Plateau border when no fibre is present.

Fig. 8(a) shows, for our SE simulation, the effect of moving the fibre in the positive $x$ direction, away from its initial central position: the Plateau border on the leading edge lengthens while those on the trailing edge shorten (see Fig. 8(a)). In simulation, when the displacement $x$ exceeds some 'detachment point', $x_{b} \approx 0.23$, the arrangement of films becomes unstable, prompting a topological change, as shown in Fig. 8(b). In our experiments, while the fibre is moving in the positive direction (Fig. 8(c)), this instability is reached earlier: the surface Plateau borders begin to 'pinch off' preferentially at one end, implying that this pre-empting of the transition is due to the fact that the fibre is not truly perfectly aligned with the frame. In the negative $x$ direction, the arrangement of films remains stable, even as the fibre reaches the edge of the frame. The move of fibre from the initial position changes the length of the surface Plateau border, resulting in $L_{1}>L_{2}$ (see Fig. 8(d)).

In Fig. 9 we plot the variation of $\Delta l_{1}$ and $\Delta l_{2}$ as the fibre is moved in the $x$ direction, as obtained from Surface Evolver simulations as described above, and from experiments using the setup shown in Fig. 4. The experimental results are the combination of two runs using the same frame and fibre as before: triangle side $C=46 \mathrm{~mm}$, height $A=69 \mathrm{~mm}$, fibre diameter $1 \mathrm{~mm}$. The fibre is held in place by a clamp which allows the position of the fibre to 


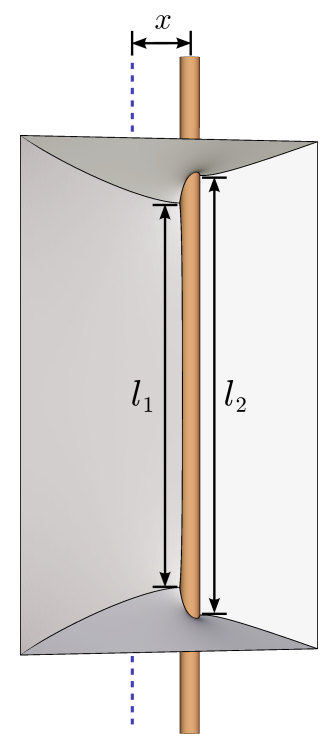

(a)

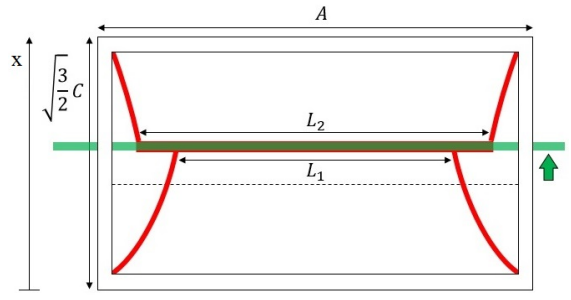

(c)

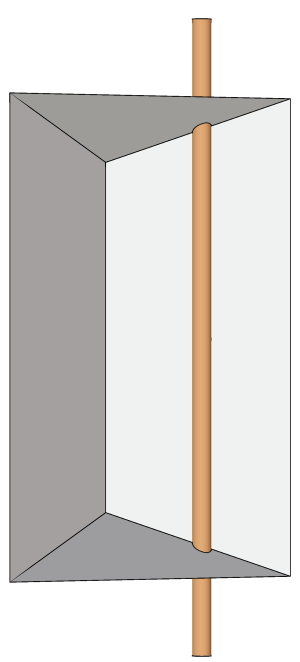

(b)

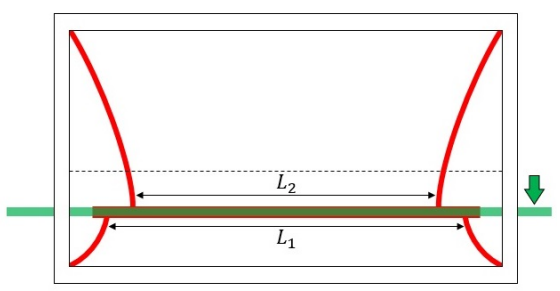

(d)

Figure 8: (a) Moving the fibre from the centre of the triangle (dashed line), to $x=0.2$ here, changes the lengths of the surface Plateau borders: $L_{1}$ decreases and $L_{2}$ increases. (b) When the fibre is moved beyond a point $x=x_{b}$ the arrangement of films becomes unstable and the fibre detaches from the Plateau border. (c) and (d) show details of the above geometry. 


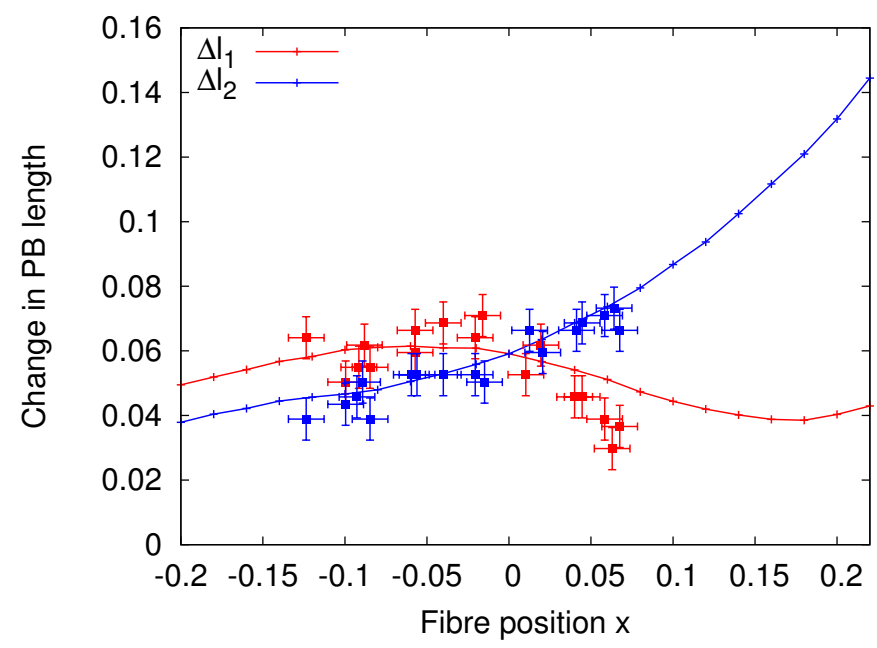

Figure 9: Variation of the normalized change in surface Plateau border lengths $\Delta l_{1}$ and $\Delta l_{2}$ as a fibre is moved along the central axis of the triangle. Position $\mathrm{x}$ is expressed in a multiple of frame side $C$. The datapoints represent experimental data; the solid lines are results from Surface Evolver simulations. Note that when $x=0, \Delta l_{1}=\Delta l_{2}$ due to symmetry.

be varied from the central position by $\pm 2 \mathrm{~cm}$ with an accuracy of $\pm 0.25 \mathrm{~mm}$.

The normalized length $l_{2}=L_{2} / C$, the length of the 'leading edge' as in Fig. 8(a), increases monotonically with $x$ : the simulation describes this variation to within experimental error over the full range of motion of the fibre. Generally, $l_{1}$ tends to decrease as the fibre is moved away from its central position. Over most of the range of $x$ we see a good approximation of $l_{1}$; however, it is systematically overestimated for large $x$; in the experiment the films peel off earlier from the fibre than in the simulation.

This discrepancy concerning $l_{1}$ between experiments and simulations when the fibre reaches the largest values of $x$ is due to the difficulty in measuring the attachment point. Indeed the lower films (see Fig. 4) become bent when the fibre is moving up, resulting in optical reflections around it.

The simulation data also shows an 'uptick' for very large $x$, near $x_{b}$. We have not been able to see this effect in experiment, once again due to the 


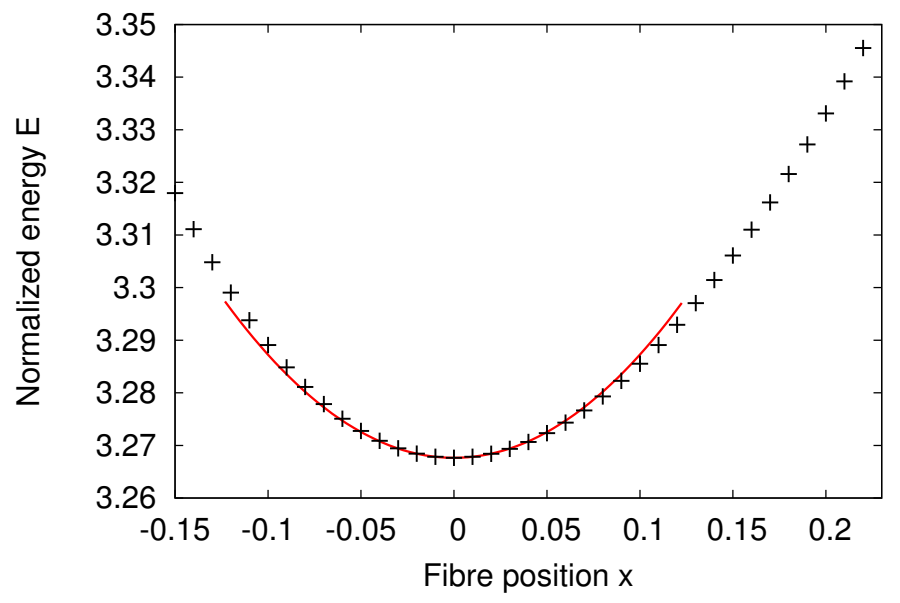

Figure 10: Variation of the total normalized surface area $E$ of the system with fibre position normalized by frame side $C$. The points are data obtained from Surface Evolver; the line is a quadratic fit for small $x$. The energy takes a minimum at $x=0$ meaning that the system is in equilibrium when the fibre is in its central position.

peeling off of the films.

\subsection{Pinning of Plateau borders to fibres}

Having convinced ourselves that Surface Evolver has been successful in modelling the shape of the films as they interact with fibres in the cases we examined, (sections 3.1 and 3.2) we now use the software to compute the area of the soap films in the presence of fibres. Note that this quantity is not obtainable from the experimental images since the films are curved. We define a dimensionless energy $E$ as the total surface area, normalized by $C^{2}$, where $C$ is the side length of the triangle.

In Fig. 10 we plot the variation of dimensionless energy $E$ with fibre position $x$. The minimum value of $E$ is achieved at $x=0$, meaning that force acts to pull the fibre back towards its central position when it is perturbed in the $x$ direction. The Plateau border is effectively pinned to the fibre, up to a displacement $x_{b}$, where it detaches. 
Such pinning has recently been suggested as being responsible for the slow down in coarsening in foams containing cellulose fibres [5] with a similar effect also for $2 \mathrm{~d}$ foams [15]. For bubbles to evolve due to inter-bubble gas diffusion, motion of films and Plateau borders is necessary. If such motion is impeded due to the presence of pinning centres, coarsening will slow down.

Using our results from the Surface Evolver calculation we can estimate whether the pinning of the Plateau borders is sufficient to slow-down coarsening. For small fibre displacement $x$ we have $E(x) \approx 3.27+1.96 x^{2}$. The force required to peel off the fibre (as shown in Fig. 8(b)) is thus given by $C \gamma d E /\left.d x\right|_{x_{b}} \simeq \gamma C$ for our break-off point $x_{b} \approx 0.23$, surface tension $\gamma$ and frame size $C$.

We can now compare this magnitude of the force to the forces experienced as a result of coarsening. We know that the Laplace pressure across the surface of a bubble is $\Delta p=4 \gamma / R$ where $R$ is the bubble radius. If we approximate the area of the contact as $1 / 13$ the surface area of the bubble (using here the often-cited 'ideal' number of contacts $\bar{z} \approx 13.397$, as obtained by considering the solid angle of the vertex of a tetrahedron), we obtain a corresponding force $F=\frac{4 \gamma}{R} \cdot \frac{4 \pi R^{2}}{13} \approx 4 \gamma R$. Our simulations gave a value for the force of about $C \gamma$ for the force necessary for detachment of the fibre. We may assume that $C$ (the side length of the triangle in the experiment) is of the same order of magnitude as $R$.

This suggests that the local force due to coarsening is greater than, but of a similar order of magnitude to, the force necessary to detach the fibre from a Plateau border. This would suggest that there is indeed some relevant degree of 'pinning' of Plateau borders to fibres in a fibre-laden foam, to the extent that the rate of coarsening is impeded, but not prevented entirely. This is in line with what has been observed in experiments [5]. 


\section{Conclusion}

We have shown that the introduction of a fibre into an arrangement of soap films alters its geometry from the equilibrium configuration as described by Plateau's laws. The response of the soap films to the presence of a fibre in both a film and a Plateau border can be accurately modeled using the Surface Evolver, at least in the simple model systems presented here.

When a fibre introduced into a Plateau border is moved, the effect is that the Plateau border tends to move with it; in other words, the Plateau border is pinned to the fibre until it pinches off at a large enough displacement. This provides a possible explanation of the observed slowdown of coarsening in foams containing fibres.

\section{Acknowledgements}

This publication has emanated from research supported in part by a research grant from Science Foundation Ireland (SFI) under [grant number 13/IA/1926]. We also acknowledge the support of the MPNS COST Actions MP1106 "Smart and green interfaces" and MP1305 "Flowing matter" and the European Space Agency [AO-09-943, AO-99-108, AO-09-813]. We thank project student D. McDermott for some experimental contributions to this work.

\section{A Details of Surface Evolver Simulations}

Given an initial non-minimized arrangement of films and boundaries, the Surface Evolver software [8] approximates the surfaces as fine triangular meshes, and via a gradient descent method finds a minimum energy configuration.

Fig. 11 (a) shows the initial configuration of facets input into Surface Evolver for the quasi-2D setup. Edges which lie on the top plate are con- 
strained to remain within that plane, and similarly for the bottom plate. The edges located at the pins are fixed in place. The shaded facet is assigned a surface tension of zero to represent the absence of a soap film in the region between the plates spanned by the fibre; all other surfaces have tension 1 . The top edge of the shaded facet is also constrained to remain at its assigned height.

(a)

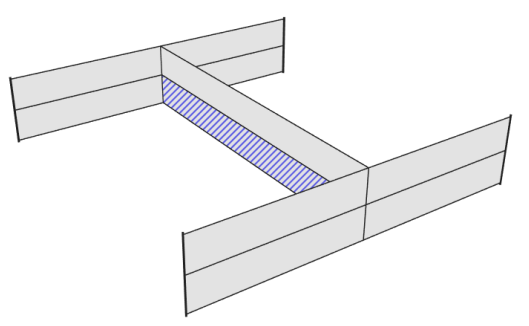

(b)

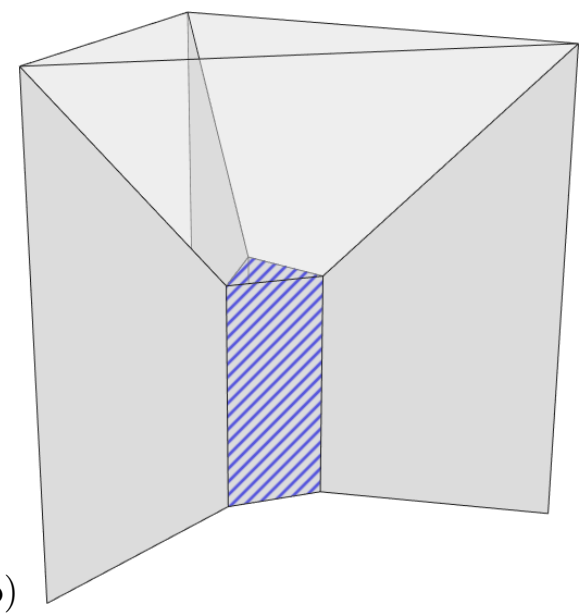

Figure 11: Initial configuration of films for (a) the quasi-2D simulation and (b) equilateral triangular prism. The shaded films have been assigned a surface tension of zero. The upper films have been rendered semi-transparent for ease of visualization.

For the simulation of a single Plateau border we exploit a symmetry of the experiment. The setup has reflectional symmetry in the direction of the axis of the prism, and so we only need to simulate half of the films.

Fig. 11 (b) shows the initial configuration of facets we used in this case. The fibre is represented initially by three facets of surface tension zero which are constrained to lie within a cylindrical shell, whose radius is specified to match the radius of the fibre in the experiment, and whose position can be varied in the $x$ direction, in line with the experiment. The edges which represent the wires of the wire frame are fixed in place, and those edges which lie in the plane of symmetry (the bottom, as viewed here) we constrain to 
remain in that plane.

Here we use the quadratic model, in which edges are represented by quadratic splines. We found that the default linear model resulted in much noisier $l_{1}(x)$ and $l_{2}(x)$ data as the fibre was moved, despite attaining energies very close to the quadratic case.

\section{References}

[1] MK Smith, VW Punton, and AG Rixson. The structure and properties of paper formed by a foaming process. Tappi, 57(1):107-111, 1974.

[2] AM Al-Qararah, T Hjelt, A Koponen, A Harlin, and Jukka A Ketoja. Bubble size and air content of wet fibre foams in axial mixing with macro-instabilities. Colloids and Surfaces A: Physicochemical and Engineering Aspects, 436:1130-1139, 2013.

[3] Ahmad M Al-Qararah, Tuomo Hjelt, Antti Koponen, Ali Harlin, and Jukka A Ketoja. Response of wet foam to fibre mixing. Colloids and Surfaces A: Physicochemical and Engineering Aspects, 467:97-106, 2015 .

[4] AM Al-Qararah, A Ekman, T Hjelt, JA Ketoja, H Kiiskinen, A Koponen, and J Timonen. A unique microstructure of the fiber networks deposited from foam-fiber suspensions. Colloids and Surfaces A: Physicochemical and Engineering Aspects, 482:544-553, 2015.

[5] B Haffner, FF Dunne, S Burke, and S Hutzler. Ageing of fibre-laden aqueous foams. Cellulose, 2016 (accepted).

[6] Majid Alimadadi and Tetsu Uesaka. 3d-oriented fiber networks made by foam forming. Cellulose, 23(1):661-671, 2016.

[7] Christopher JS Petrie. The rheology of fibre suspensions. Journal of Non-Newtonian Fluid Mechanics, 87(2):369-402, 1999. 
[8] KA Brakke. The Surface Evolver. Experimental mathematics, 1(2):141$165,1992$.

[9] Joseph Antoine Ferdinand Plateau. Statique expérimentale et théorique des liquides soumis aux seules forces moléculaires, volume 2. GauthierVillars, 1873.

[10] D Weaire and S Hutzler. The Physics of Foams. Oxford University Press, 2001.

[11] I Cantat, S Cohen-Addad, F Elias, F Graner, R Höhler, R Flatman, O Pitois, F Rouyer, A Saint-Jalmes, and Editor: Simon Cox. Foams: Structure and Dynamics. Oxford University Press, 2013.

[12] M In Het Panhuis, S Hutzler, D Weaire, and R Phelan. New variations on the soap film experiments of plateau i. experiments under forced drainage. Philosophical Magazine B, 78(1):1-12, 1998.

[13] DGT Barrett, S Kelly, EJ Daly, MJ Dolan, W Drenckhan, D Weaire, and S Hutzler. Taking plateau into microgravity: The formation of an eightfold vertex in a system of soap films. Microgravity-Science and Technology, 20(1):17-22, 2008.

[14] Caroline A Schneider, Wayne S Rasband, and Kevin W Eliceiri. NIH Image to ImageJ: 25 years of image analysis. Nat methods, 9(7):671$675,2012$.

[15] O Krichevsky and J Stavans. Coarsening of two-dimensional soap froths in the presence of pinning centers. Physical Review B, 46(17):10579, 1992. 


\begin{abstract}
We present experiments and Surface Evolver simulations concerning the interaction of fibres with simple arrangements of soap films, which constitute model systems for dry foams. For a fibre inserted into a soap film which spans two Plateau borders, our simulation accurately predicts the variation in the length of the film as the fibre thickness is varied. For a fibre introduced into a Plateau border, simulations accurately predict the variation in length of the Plateau border as the fibre diameter is varied, and as it is moved. We suggest that the force necessary to move the fibre from its equilibrium position may act to inhibit foam coarsening, in line with previous observations from experiments on fibre-laden foams.
\end{abstract}

